\title{
Generalized Power Akshaya Distribution and Its Applications
}

\author{
Ahmed T. Ramadan', Ahlam H. Tolba², Beih S. El-Desouky ${ }^{2}$ \\ ${ }^{1}$ Department of Basic Sciences, High Raya Institute, New Damietta, Egypt \\ ${ }^{2}$ Department of Mathematics, Faculty of Science, Mansoura University, Mansoura, Egypt \\ Email: atramadan88@gmail.com,Dr_ahamdy156@mans.edu.eg,b_desouky@yahoo.com
}

How to cite this paper: Ramadan, A.T., Tolba, A.H. and El-Desouky, B.S. (2021) Generalized Power Akshaya Distribution and Its Applications. Open Journal of Modelling and Simulation, 9, 323-338. https://doi.org/10.4236/ojmsi.2021.94021

Received: June 30, 2021

Accepted: August 27, 2021

Published: August 30, 2021

Copyright $\odot 2021$ by author(s) and Scientific Research Publishing Inc. This work is licensed under the Creative Commons Attribution International License (CC BY 4.0).

http://creativecommons.org/licenses/by/4.0/

\begin{abstract}
In this paper, a new two-parameter distribution called generalized power Akshaya distribution extended from Akshaya distribution is introduced. This distribution is proposed to model lifetime data. Statistical properties like density, hazard, survival and moments are derived. Two parameters estimation is introduced using maximum likelihood and Bayesian techniques. Finally, an application of real data and a simulation study are introduced to illustrate the usefulness of the proposed distribution.
\end{abstract}

\section{Keywords}

Akshaya Distribution, Generalized Distributions, Moments, Estimation

\section{Introduction}

The statistical analysis and modeling of lifetime data are necessary for many applied sciences like insurance, finance, bio-medical and engineering sciences. So, many lifetime distributions are introduced in the last era. Al-Kutubi et al. [1] presented the Properties and estimation methods of a new extended two-parameter distribution and its applications in medicine and geology. Also, Brooks and Steven [2] provided a comprehensive tutorial review about Markov chain Monte Carlo (MCMC) algorithms and discussed some implementation issues associated with MCMC methods. Eliwa et al. [3] proposed a new generator of distributions and some of its fundamental properties. Epstein et al. [4] discussed statistical problems which arise when the observations become available in an ordered manner. Also in 2013 Ghitany et al. [5] introduced the statistical properties of new twoparameter distribution called power Lindley mixed of Weibull and generalized gamma distributions. In addition, Ghitany et al. [6] showed that Lindley distribution is a better model than the exponential distribution. In 1980, Glaser [7] 
studied the sufficient conditions that ensure that a lifetime density has an increasing, decreasing or a bathtub-shaped failure rate. Measuring skewness and a quantile alternative for kurtosis are studied in [8] and [9] respectively. In 2011, Nadarajah et al. [10] introduced the generalized Lindley distribution and proved that it is better than gamma, lognormal, Weibull and exponential distributions with bathtub hazard rate. Okasha and Mustafa [11] used the E-Bayesian estimation for the Weibull distribution based on adaptive progressive hybrid censored competing risks. Rama Shanker [12] introduced a new one parameter Akash distribution which is a mixture of exponential $(\theta)$ and gamma $(3, \theta)$ distributions. Also, he introduced Akshaya distribution with one parameter [13] for modeling lifetime data which gave a better fit than the classical exponential and Lindley distributions. Shanker et al. [14] presented the relationships and comparative studies of Akash, Shanker, Lindley and exponential distributions and estimated their parameters. Besides that the previous articles talked about some lifetime distributions, other articles talked about parameters estimation methods. Finally, Smith et al. [15] developed and compared the maximum likelihood and Bayesian estimators for the three-parameter Weibull distribution.

According to Shanker [13], the probability density function (pdf) of Akshaya distribution is given by

$$
f(x ; \theta)=\frac{\theta^{4}}{\theta^{3}+3 \theta^{2}+6 \theta+6}(1+x)^{3} \mathrm{e}^{-\theta x}, x, \theta>0,
$$

the cumulative distribution function (CDF) is given by

$$
F(x ; \theta)=1-\left\{1+\frac{\theta^{3} x^{3}+3 \theta^{2}(\theta+1) x^{2}+3 \theta\left(\theta^{2}+2 \theta+2\right) x}{\theta^{3}+3 \theta^{2}+6 \theta+6}\right\} \mathrm{e}^{-\theta x}, x, \theta>0,
$$

and the hazard rate function is given by

$$
h(x ; \theta)=\frac{\theta^{4}(1+x)^{3}}{\theta^{3} x^{3}+3 \theta^{2}(\theta+1) x^{2}+3 \theta\left(\theta^{2}+2 \theta+2\right) x+\left(\theta^{3}+3 \theta^{2}+6 \theta+6\right)}, x, \theta>0 .
$$

The hazard rate function given in Equation (3) is increasing function of $x$ and $\theta$. However, Akshaya distribution is not suitable for many situations from a theoretical point of view. So, a more flexible extension of Akshaya distribution is introduced in this paper.

Ghitany et al. [5] used the transformation $X=Y^{\frac{1}{\alpha}}$ to generate a new distribution called power Lindley distribution. By using this transformation, a new generalized power Akshaya distribution can be introduced.

$$
\begin{aligned}
& \text { Let } \begin{array}{rl}
y=x^{\alpha} \rightarrow x=y^{\frac{1}{\alpha}} \rightarrow \mathrm{d} & x=\frac{1}{\alpha} y^{\frac{1}{\alpha}-1} \mathrm{~d} y \\
& \quad F(y)=F_{0}\left(x^{\alpha}\right), f(y)=\alpha x^{\alpha-1} f_{0}\left(x^{\alpha}\right) .
\end{array}
\end{aligned}
$$

The aim of this paper is to study some properties of the generalized power Akshaya distribution including the density and hazard functions as in Section 2. Section 3 studied some statistical properties like moments of the distribution, 
incomplete moments, mean residual lifetime and mean time to failure. Two methods of parameter estimation are given in Section 4. Application of two types of data, real data and simulation study are presented in Section 5 to show the flexleibility of the distribution.

\section{Generalized Power Akshaya Distribution}

\section{Some Basic Functions}

According to Equations (1), (2) and (4), the cumulative distribution function (CDF) and the probability density function (pdf) of power Akshaya distribution are given respectively as

$$
\begin{gathered}
F(x ; \theta, \alpha)=1-\left\{1+\frac{\theta^{3} x^{3 \alpha}+3 \theta^{2}(\theta+1) x^{2 \alpha}+3 \theta\left(\theta^{2}+2 \theta+2\right) x^{\alpha}}{\theta^{3}+3 \theta^{2}+6 \theta+6}\right\} \mathrm{e}^{-\theta x^{\alpha}}, x, \theta, \alpha>0, \\
f(x ; \theta, \alpha)=\frac{\alpha x^{\alpha-1} \theta^{4}}{\theta^{3}+3 \theta^{2}+6 \theta+6}\left(1+x^{\alpha}\right)^{3} \mathrm{e}^{-\theta x^{\alpha}}, \theta, \alpha>0
\end{gathered}
$$

Akshaya distribution function is obtained from Equation (5) when $\alpha=1$.

From Equation (6), we can notice the behavior of $f(x ; \theta, \alpha)$ at $x=0$ and $x=\infty$ as the following

$$
f(0)=\left\{\begin{array}{l}
\infty, \quad \alpha<1, \\
\frac{\theta^{4}}{\theta^{3}+3 \theta^{2}+6 \theta+6}, \quad \alpha=1, \quad f(\infty)=0 . \\
0, \quad \alpha>1,
\end{array}\right.
$$

The following theorem shows that there are three shapes for the density function of the generalized power Akshaya distribution according to the values of the parameters $\theta$ and $\alpha$.

Theorem 1. The density function of the generalized power Akshaya distribution given in Equation (6) is

(a) decreasing if $0<\alpha \leq 1, \theta \geq \eta_{1}$,

(b) uni-modal if $\alpha \geq 1$,

(c) decreasing-increasing-decreasing if $0<\alpha \leq 1,0<\theta<\eta_{1}$,

Where $\eta_{1}=\frac{1+2 \alpha-2 \sqrt{3 \alpha(1-\alpha)}}{\alpha}$.

Proof. The first derivative of $f(x ; \theta, \alpha)$ of generalized power Akshaya distribution is

$$
f^{\prime}(x)=\frac{\alpha \theta^{4}}{\theta^{3}+3 \theta^{2}+6 \theta+6} x^{\alpha-2}\left(1+x^{\alpha}\right)^{2} \mathrm{e}^{-\theta x^{\alpha}} \Psi_{1}\left(x^{\alpha}\right), x>0,
$$

where $\Psi_{1}(y)=a_{1} y^{2}+b_{1} y+c_{1}, y=x^{\alpha}>0$, and $a_{1}=-\theta \alpha, b_{1}=4 \alpha-1-\theta \alpha, c_{1}=\alpha-1$.

It is obvious that $f^{\prime}(x)$ and $\Psi_{1}(y)$ have the same sign. The function $\Psi_{1}(y)$ is:

(a) decreasing if $\Psi_{1}$ has one or no real roots, 


$$
b_{1}^{2}-4 a_{1} c_{1} \leq 0 \text {, then }(4 \alpha-1-\theta \alpha)^{2}+4 \theta \alpha(\alpha-1) \leq 0
$$

which implies to $0<\alpha \leq 1, \theta \geq \frac{1+2 \alpha-2 \sqrt{3 \alpha(1-\alpha)}}{\alpha}$,

with $\Psi_{1}(0)=c_{1}, \Psi_{1}(\infty)=-\infty$.

(b) uni-modal with maximum value at the point $y_{1}=\frac{-b_{1}}{2 a_{1}}$ if $c_{1}>0$, i.e. $\alpha>1$.

(c) combining stated conditions in (a) and (b) we note that the function $\Psi_{1}(y)$ changes its sign from negative to positive to negative and this completes the proof.

Figure 1 and Figure 2 show the $\mathrm{CDF}$ and pdf functions of generalized power Akshaya distribution for different values of $\theta, \alpha$.

The survival function, $S(x)$ and the hazard function, $H(x)$ of generalized power Akshaya distribution, are given respectively as

$$
\begin{aligned}
& S(x ; \theta, \alpha)=1-F(x ; \theta, \alpha) \\
& =\left\{1+\frac{\theta^{3} x^{3 \alpha}+3 \theta^{2}(\theta+1) x^{2 \alpha}+3 \theta\left(\theta^{2}+2 \theta+2\right) x^{\alpha}}{\theta^{3}+3 \theta^{2}+6 \theta+6}\right\} \mathrm{e}^{-\theta x^{\alpha}}, x, \theta, \alpha>0,
\end{aligned}
$$

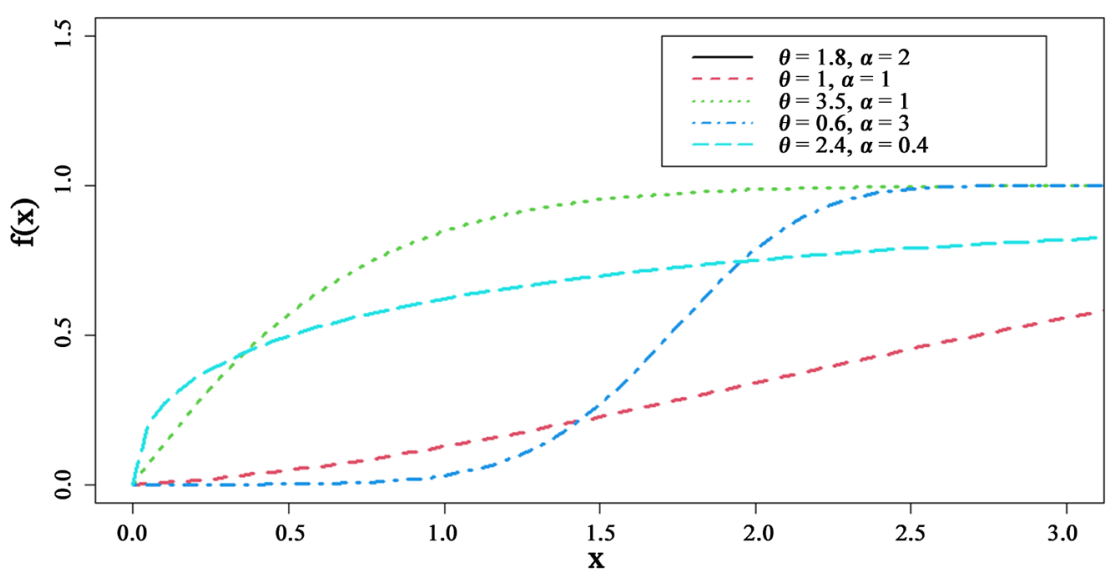

Figure 1. CDF of generalized power Akshaya distribution.

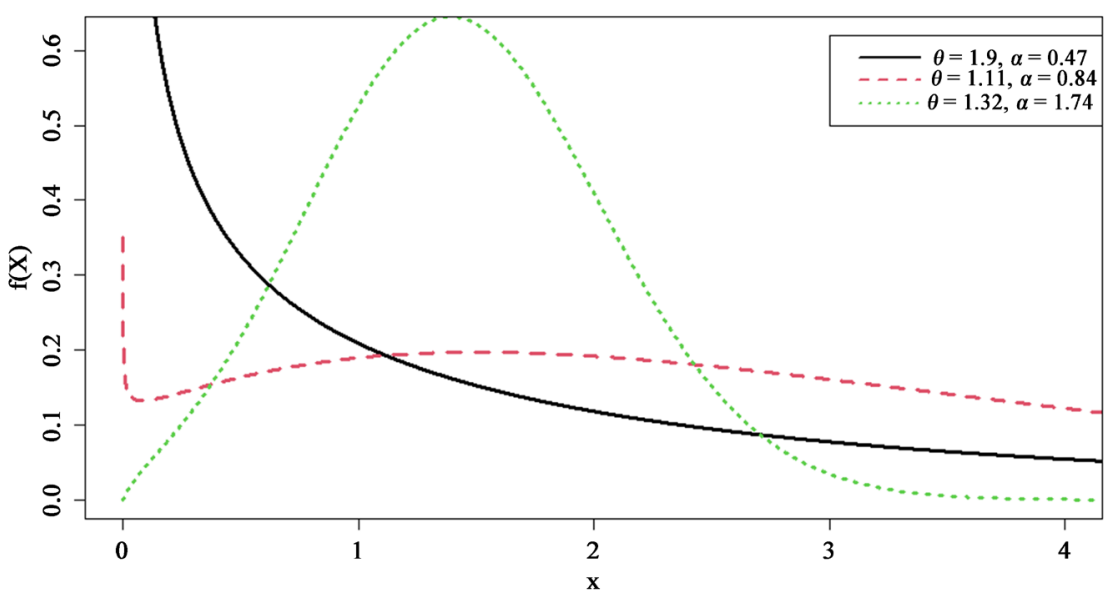

Figure 2. Pdf of generalized power Akshaya Distribution. 


$$
\begin{aligned}
& H(x ; \theta, \alpha)=\frac{f(x ; \theta, \alpha)}{S(x ; \theta, \alpha)} \\
& =\frac{\alpha \theta^{4} x^{\alpha-1}\left(1+x^{\alpha}\right)^{3}}{\theta^{3}\left(1+x^{3 \alpha}\right)+3 \theta^{2}\left(1+(\theta+1) x^{2 \alpha}\right)+3 \theta\left(2+\left(\theta^{2}+2 \theta+2\right) x^{\alpha}\right)+6}, x, \theta, \alpha>0 .
\end{aligned}
$$

From Equation (8), we can notice that the behavior of $H(x ; \theta, \alpha)$ at $x=0$ is the same as the behavior of $f(x ; \theta, \alpha)$ at $x=0$, so that

$$
H(0)=\left\{\begin{array}{l}
\infty, \quad \alpha<1, \\
\frac{\theta^{4}}{\theta^{3}+3 \theta^{2}+6 \theta+6}, \quad \alpha=1, \\
0, \quad \alpha>1,
\end{array}\right.
$$

and

$$
H(\infty)= \begin{cases}0, & \alpha<1, \\ \theta, & \alpha=1, \\ \infty, & \alpha>1 .\end{cases}
$$

The analytic analysis of the hazard function shape is very complicated, so according to [7], Glaser's theorem is applied here. Now,

$$
\begin{gathered}
\eta(x)=-\frac{f^{\prime}(x)}{f(x)}=\frac{\alpha \theta x^{2 \alpha}+x^{\alpha}(\alpha(\theta-4)+1)+1-\alpha}{x\left(1+x^{\alpha}\right)}, \\
\eta^{\prime}(x)=\frac{\Psi_{2}(x)}{x^{2}\left(1+x^{\alpha}\right)^{2}},
\end{gathered}
$$

where

$$
\begin{aligned}
\Psi_{2}(x)= & \alpha \theta(\alpha-1) x^{3 \alpha}+x^{2 \alpha}\left(2 \theta \alpha^{2}-2 \alpha(\theta-2)-1\right) \\
& +x^{\alpha}(\alpha-1)(\alpha(\theta-3)+2)+\alpha-1 .
\end{aligned}
$$

Consequently, the hazard function, $H(x)$, and the function $\Psi_{2}(x)$ have the same sign and the function $\Psi_{2}(x)$ is:

(a) increasing if $\alpha \geq 1, \theta>0$,

(b) decreasing if $0<\alpha \leq 0.25, \theta>0$,

(c) decreasing-increasing-decreasing if $0.25<\alpha<1, \theta>0$.

Figure 3 and Figure 4 show the survival and hazard functions of generalized power Akshaya distribution for different values of $\theta, \alpha$.

\section{Statistical Properties}

\subsection{Moments}

In this subsection, the first four moments about zero and about mean and the incomplete moments of generalized power Akshaya distribution are derived. The general form of the $r^{\text {th }}$ moment about zero is given by

$$
\mu_{r}^{\prime}=E\left(x^{r}\right)=\int_{0}^{\infty} x^{r} f(x ; \theta, \alpha) \mathrm{d} x .
$$




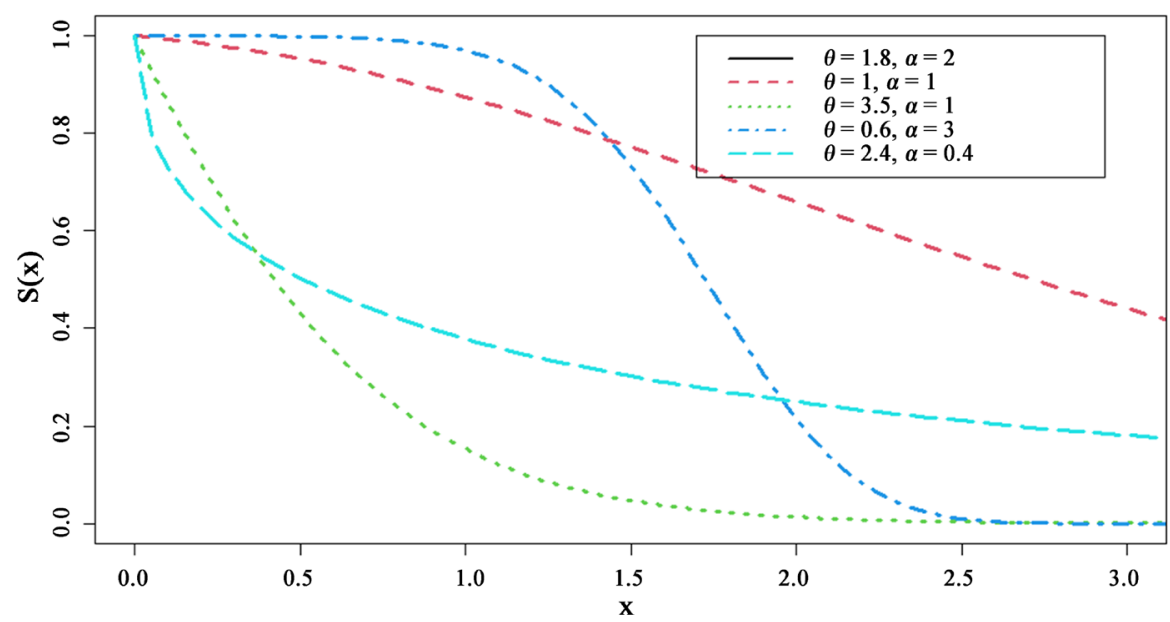

Figure 3. Survival function of generalized power Akshaya distribution.

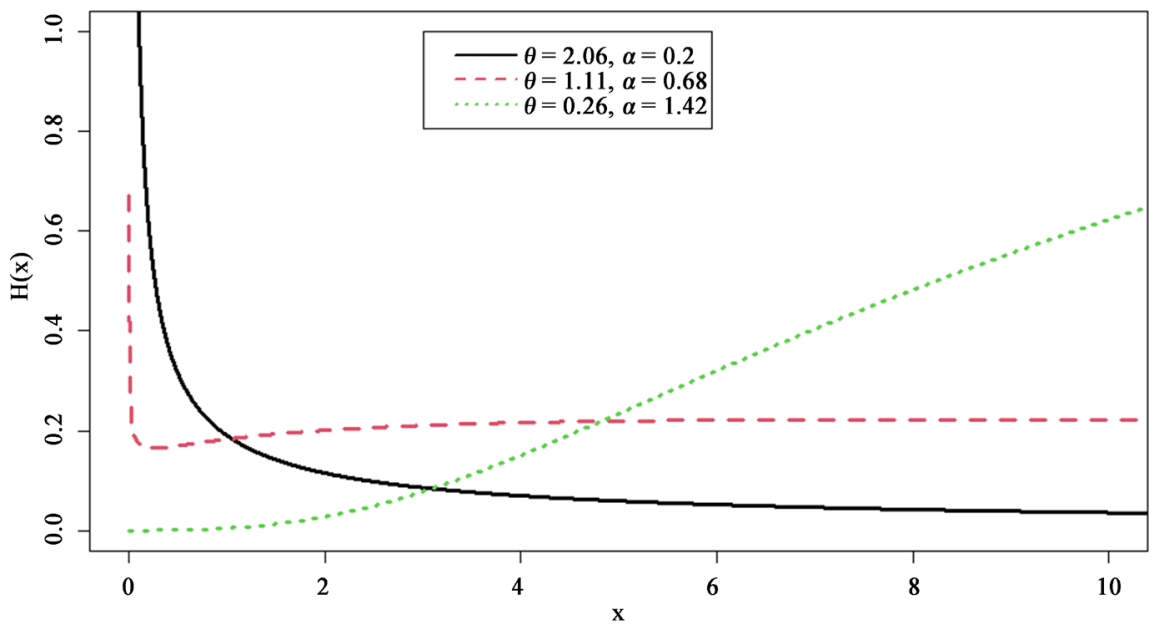

Figure 4. Hazard function of generalized power Akshaya distribution.

According to Equation (9) and using Equation (6), the $r^{\text {th }}$ moment about zero of generalized power Akshaya distribution is given by

$$
\mu_{r}^{\prime}=c \alpha \int_{0}^{\infty} x^{r} x^{\alpha-1}\left(1+x^{\alpha}\right)^{3} \mathrm{e}^{-\theta x^{\alpha}} \mathrm{d} x, c=\frac{\theta^{4}}{\theta^{3}+3 \theta^{2}+6 \theta+6} .
$$

Let $t=\theta x^{\alpha} \rightarrow x=\left(\frac{t}{\theta}\right)^{\frac{1}{\alpha}} \rightarrow \mathrm{d} x=\frac{1}{\theta \alpha}\left(\frac{t}{\theta}\right)^{\frac{1}{\alpha}-1} \mathrm{~d} t$,

$$
\mu_{r}^{\prime}=c \alpha \int_{0}^{\infty}\left(\frac{t}{\theta}\right)^{\frac{r}{\alpha}}\left(\frac{t}{\theta}\right)^{1-\frac{1}{\alpha}}\left(1+\left(\frac{t}{\theta}\right)\right)^{3} \mathrm{e}^{-\theta\left(\frac{t}{\theta}\right)} \frac{1}{\theta \alpha}\left(\frac{t}{\theta}\right)^{\frac{1}{\alpha}-1} \mathrm{~d} t .
$$

Finally, the $r^{\text {th }}$ moment about zero of generalized power Akshaya distribution is given by

$$
\mu_{r}^{\prime}=\frac{r \theta^{\frac{-r}{\alpha}}\left(r^{3}+3 r^{2} \alpha(2+\theta)+r \alpha^{2}(11+3 \theta(3+\theta))+\alpha^{3}(6+\theta(6+\theta(3+\theta)))\right) \Gamma\left(\frac{r}{\alpha}\right)}{\alpha^{4}(6+\theta(6+\theta(3+\theta)))} .
$$


Substituting in Equation (10) with $r=1,2,3$ and 4 we get

$$
\begin{gathered}
\mu_{1}^{\prime}=\frac{\theta^{\frac{-1}{\alpha}}(1+\alpha(3(2+\theta)+\alpha(11+3 \theta(3+\theta)+\alpha(6+\theta(6+\theta(3+\theta)))))) \Gamma\left(\frac{1}{\alpha}\right)}{\alpha^{4}(6+\theta(6+\theta(3+\theta)))}, \\
\mu_{2}^{\prime}=\frac{2 \theta^{\frac{-2}{\alpha}}(8+\alpha(12(2+\theta)+\alpha(22+6 \theta(3+\theta)+\alpha(6+\theta(6+\theta(3+\theta)))))) \Gamma\left(\frac{2}{\alpha}\right)}{\alpha^{4}(6+\theta(6+\theta(3+\theta)))}, \\
\mu_{3}^{\prime}=\frac{3 \theta^{\frac{-3}{\alpha}}(27+\alpha(27(2+\theta)+\alpha(33+9 \theta(3+\theta)+\alpha(6+\theta(6+\theta(3+\theta)))))) \Gamma\left(\frac{3}{\alpha}\right)}{\alpha^{4}(6+\theta(6+\theta(3+\theta)))},
\end{gathered}
$$

and

$$
\mu_{4}^{\prime}=\frac{4 \theta^{\frac{-4}{\alpha}}\left(64+48 \alpha(2+\theta)+4 \alpha^{2}(11+3 \theta(3+\theta))+\alpha^{3}(6+\theta(6+\theta(3+\theta)))\right) \Gamma\left(\frac{4}{\alpha}\right)}{\alpha^{4}(6+\theta(6+\theta(3+\theta)))} .
$$

Also, the first four moments about mean can be deduced from moments about zero as follows

$$
\begin{gathered}
\mu_{1}=0, \\
\mu_{2}=\mu_{2}^{\prime}-\left(\mu_{1}^{\prime}\right)^{2}, \\
\mu_{3}=\mu_{3}^{\prime}-3 \mu_{2}^{\prime} \mu_{1}^{\prime}+2\left(\mu_{1}^{\prime}\right)^{3} \text { and } \\
\mu_{4}=\mu_{4}^{\prime}-4 \mu_{3}^{\prime} \mu_{1}^{\prime}+6 \mu_{2}^{\prime}\left(\mu_{1}^{\prime}\right)^{2}-3\left(\mu_{1}^{\prime}\right)^{4} .
\end{gathered}
$$

\subsection{Incomplete Moments and Related Measures}

In this subsection, we introduce the $r^{\text {th }}$ incomplete moment, $m_{r}(y)$ and some related measures like mean deviation about mean and median and Bonferroni and Lorenz curves.

\subsubsection{Incomplete Moments}

The $r^{\text {th }}$ incomplete moments is given by

$$
\begin{aligned}
m_{r}(y)= & \int_{0}^{y} x^{r} f(x) \mathrm{d} x \\
= & \frac{y^{r}\left(\theta y^{\alpha}\right)^{\frac{-r}{\alpha}}}{6+6 \theta+3 \theta^{2}+\theta^{3}}\left(\theta^{3} \Gamma\left(1+\frac{r}{\alpha}\right)+3 \theta^{2} \Gamma\left(2+\frac{r}{\alpha}\right)+3 \theta \Gamma\left(3+\frac{r}{\alpha}\right)\right. \\
& +\Gamma\left(4+\frac{r}{\alpha}\right)-\theta^{3} \Gamma\left(1+\frac{r}{\alpha}, \theta y^{\alpha}\right)-3 \theta^{2} \Gamma\left(2+\frac{r}{\alpha}, \theta y^{\alpha}\right) \\
& \left.-3 \theta \Gamma\left(3+\frac{r}{\alpha}, \theta y^{\alpha}\right)-\Gamma\left(4+\frac{r}{\alpha}, \theta y^{\alpha}\right)\right) .
\end{aligned}
$$

\subsubsection{Mean Deviation about Mean and Median}

Mean deviation about mean of a parameter $X \sim$ power Akshaya distribution, $\delta_{1}(x)$ can be given as follows 


$$
\begin{aligned}
\delta_{1}(x) & =\int_{0}^{\infty}\left|x-\mu_{1}^{\prime}\right| f(x) \mathrm{d} x \\
& =2 \mu_{1}^{\prime} F\left(\mu_{1}^{\prime}\right)-2 \int_{0}^{\mu_{1}^{\prime}} x f(x) \mathrm{d} x \\
& =2 \mu_{1}^{\prime} F\left(\mu_{1}^{\prime}\right)-2 m_{1}\left(\mu_{1}^{\prime}\right),
\end{aligned}
$$

where $m_{1}($.$) is the incomplete moment given in Equation (11) when r=1$.

The mean deviation about median $(M)$ of a parameter $X \sim$ power Akshaya distribution, $\delta_{2}(x)$ can be given as follows

$$
\begin{aligned}
\delta_{2}(x) & =\int_{0}^{\infty}|x-M| f(x) \mathrm{d} x \\
& =\mu_{1}^{\prime}-2 \int_{0}^{M} x f(x) \mathrm{d} x \\
& =\mu_{1}^{\prime}-2 m_{1}(M) .
\end{aligned}
$$

\subsubsection{Bonferroni and Lorenz Curves}

The Bonferroni and Lorenz curves have large applications in economy to study income and poverty and other fields. Bonferroni and Lorenz curves are defined as

$$
\begin{aligned}
B(p) & =\frac{1}{p \mu} \int_{0}^{q} x f(x) \mathrm{d} x \\
& =\frac{m_{1}(q)}{p \mu}, \\
L(p) & =\frac{1}{\mu} \int_{0}^{q} x f(x) \mathrm{d} x \\
& =\frac{m_{1}(q)}{\mu} .
\end{aligned}
$$

\subsection{Quantile Function, Bowley Skewness and Moors Kurtosis}

For any $q \in(0,1)$, the $q^{\text {th }}$ quantile function $(\mathrm{Q}(\mathrm{q}))$ is the solution of $F(Q(q))=q ; Q(q)>0$, in other words, $q=F^{-1}(x ; \theta, \alpha)$.

It's obvious if we set $q=0.5$ we get the median $(M)$. Bowley skewness [8] and Moors kurtosis [9] can be obtained as

$$
\text { Bowley skewness }=\frac{Q\left(\frac{3}{4}\right)+Q\left(\frac{1}{4}\right)-2 Q\left(\frac{1}{2}\right)}{Q\left(\frac{3}{4}\right)-Q\left(\frac{1}{4}\right)},
$$

and

$$
\text { Moors kurtosis }=\frac{Q\left(\frac{3}{8}\right)-Q\left(\frac{1}{8}\right)+Q\left(\frac{7}{8}\right)-Q\left(\frac{5}{8}\right)}{Q\left(\frac{6}{8}\right)-Q\left(\frac{2}{8}\right)} \text {. }
$$

\subsection{Mean Residual Lifetime}

Mean residual lifetime $(m)$ is a reliability term based on lifetime of the product. It is a way to give a numeric value based on the residual lifetime of the product. 
Mean residual lifetime (m) can be given as follows

$$
\begin{aligned}
& m(x)=E[X-x / X>x]=\frac{1}{1-F(x)} \int_{x}^{\infty}(1-F(t)) \mathrm{d} t \\
& =\left[\mathrm { e } ^ { \theta x ^ { \alpha } } \theta ^ { \frac { - 1 } { \alpha } } ( \theta x ^ { \alpha } ) ^ { \frac { - 1 } { \alpha } } ( - x \theta ^ { \frac { 1 } { \alpha } } + ( \theta x ^ { \alpha } ) ^ { \frac { 1 } { \alpha } } ) \left(1+3 \alpha(2+\theta)+\alpha^{2}\left(11+9 \theta+3 \theta^{2}\right)\right.\right. \\
& \left.+\alpha^{3}\left(6+6 \theta+3 \theta^{2}+\theta^{3}\right)\right) \Gamma\left(\frac{1}{\alpha}\right)+x \alpha^{3} \theta^{\frac{1}{\alpha}}\left(3\left(2+2 \theta+\theta^{2}\right) \Gamma\left(1+\frac{1}{\alpha}, \theta x^{\alpha}\right)\right. \\
& +3(1+\theta) \Gamma\left(2+\frac{1}{\alpha}, \theta x^{\alpha}\right)+\Gamma\left(3+\frac{1}{\alpha}, \theta x^{\alpha}\right)+6 \theta \Gamma\left(\frac{1}{\alpha}, \theta x^{\alpha}\right) \\
& \left.\left.+3 \theta^{2} \Gamma\left(\frac{1}{\alpha}, \theta x^{\alpha}\right)+\theta^{3} \Gamma\left(\frac{1}{\alpha}, \theta x^{\alpha}\right)\right)\right] /\left[\alpha ^ { 4 } \left(\theta x ^ { \alpha } \left(6+3 \theta\left(2+x^{\alpha}\right)\right.\right.\right. \\
& \left.\left.\left.+\theta^{2}\left(3+3 x^{\alpha}+x^{2 \alpha}\right)\right)+\mathrm{e}^{\theta x^{\alpha}}\left(6+6 \theta+3 \theta^{2}+\theta^{3}\right)\right)\right] .
\end{aligned}
$$

\subsection{Mean Time to Failure}

Mean time to failure (MTTF) is also a reliability term based on lifetime of the product. It gives a numeric value based on a compilation of data to quantify a failure rate of the product. MTTF can be given as follows

$$
\mathrm{MTTF}=\frac{\theta^{\frac{-1}{\alpha}(1+\alpha(3(2+\theta)+\alpha(11+3 \theta(3+\theta)+\alpha(6+\theta(6+\theta(3+\theta)))))) \Gamma\left(\frac{1}{\alpha}\right)}}{\alpha^{4}\left(6+6 \theta+3 \theta^{2}+\theta^{3}\right)} .
$$

\section{Parameters Estimation}

In this section, two techniques including maximum likelihood estimation (MLE) method and Bayesian estimation method are used to estimate the parameters of generalized power Akshaya distribution.

\subsection{Maximum Likelihood Estimation Method}

Let $\left(x_{1}, x_{2}, \cdots, x_{n}\right)$ be a random sample from generalized power Akshaya distribution, then the likelihood estimation function, $\mathrm{L}$ can be given as follows

$$
L=\prod_{i=1}^{n} f(x ; \theta, \alpha)=\frac{\alpha^{n} \theta^{4 n}}{\left(\theta^{3}+3 \theta^{2}+6 \theta+6\right)^{n}} \mathrm{e}^{-\theta \sum_{i=1}^{n} x_{i}^{\alpha}} \prod_{i=1}^{n} x_{i}^{\alpha-1}\left(1+x_{i}^{\alpha}\right)^{3},
$$

and the natural log likelihood function is given by

$$
\begin{aligned}
\ln (L)= & n\left\{\ln (\alpha)+4 \ln (\theta)-\ln \left(\theta^{3}+3 \theta^{2}+6 \theta+6\right)\right\} \\
& -\theta \sum_{i=1}^{n} x_{i}^{\alpha}+\sum_{i=1}^{n}\left\{(\alpha-1) \ln \left(x_{i}\right)+3 \ln \left(1+x_{i}^{\alpha}\right)\right\} .
\end{aligned}
$$

The first derivatives of the natural log likelihood function with respect to $\theta, \alpha$ are given by

$$
\frac{\partial}{\partial \theta} \ln (L)=\frac{4 n}{\theta}-\frac{3 \theta^{2}+6 \theta+6}{\theta^{3}+3 \theta^{2}+6 \theta+6}-\sum_{i=1}^{n} x_{i}^{\alpha}
$$




$$
\frac{\partial}{\partial \alpha} \ln (L)=\frac{n}{\alpha}-\theta \sum_{i=1}^{n} x_{i}^{\alpha} \ln \left(x_{i}\right)+\sum_{i=1}^{n}\left\{\ln \left(x_{i}\right)\left(1+\frac{3 x_{i}^{\alpha}}{1+x_{i}^{\alpha}}\right)\right\} .
$$

Equations (20) and (21) have no analytic closed form when equating by zero, so numerical methods are used to give solutions. The second derivatives of the natural $\log$ likelihood function with respect to $\theta, \alpha$ can be given by

$$
\begin{gathered}
\frac{\partial^{2}}{\partial \theta^{2}} \ln (L)=\frac{-4 n}{\theta^{2}}-\frac{6(\theta+1)\left(\theta^{3}+3 \theta^{2}+6 \theta+6\right)-\left(3 \theta^{2}+6 \theta+6\right)^{2}}{\left(\theta^{3}+3 \theta^{2}+6 \theta+6\right)^{2}}, \\
\frac{\partial^{2}}{\partial \theta \partial \alpha} \ln (L)=\sum_{i=1}^{n} x^{\alpha} \ln \left(x_{i}\right), \\
\frac{\partial^{2}}{\partial \alpha \partial \theta} \ln (L)=\sum_{i=1}^{n} x^{\alpha} \ln \left(x_{i}\right), \\
\frac{\partial^{2}}{\partial \alpha^{2}} \ln (L)=\frac{-n}{\alpha^{2}}-\theta \sum_{i=1}^{n} x_{i}^{\alpha}\left(\ln \left(x_{i}\right)\right)^{2}+\sum_{i=1}^{n}\left\{\ln \left(x_{i}\right)\left(1+\frac{3 x_{i}^{\alpha} \ln \left(x_{i}\right)}{\left(1+x_{i}^{\alpha}\right)^{2}}\right)\right\} .
\end{gathered}
$$

The $(1-\zeta) 100 \%$ confidence interval for the parameters $\theta$ and $\alpha$ can be written as

$$
\left(\hat{\theta}_{L}, \hat{\theta}_{U}\right)=\hat{\theta} \mp z_{1-\frac{\zeta}{2}} \sqrt{\operatorname{var}(\hat{\theta})}, \quad\left(\hat{\alpha}_{L}, \hat{\alpha}_{U}\right)=\hat{\alpha} \mp z_{1-\frac{\zeta}{2}} \sqrt{\operatorname{var}(\hat{\alpha})},
$$

where $\hat{\theta}$ and $\hat{\alpha}$ are the maximum likelihood estimates of $\theta$ and $\alpha, z_{1-\frac{\zeta}{2}}$ is the percentile of the standard normal distribution and $\operatorname{var}(\hat{\theta}), \operatorname{var}(\hat{\alpha})$ are the asymptotic variances of maximum likelihood estimates calculated using the inverse of the information matrix as follows

$$
F^{-1}=\left[\begin{array}{ll}
\frac{-\partial^{2}}{\partial \theta^{2}} \ln L & \frac{-\partial^{2}}{\partial \theta \partial \alpha} \ln L \\
\frac{-\partial^{2}}{\partial \alpha^{2}} \ln L & \frac{-\partial^{2}}{\partial \alpha \partial \theta} \ln L
\end{array}\right]^{-1}=\left[\begin{array}{cc}
\operatorname{var}(\hat{\theta}) & \operatorname{cov}(\hat{\theta}, \hat{\alpha}) \\
\operatorname{cov}(\hat{\alpha}, \hat{\theta}) & \operatorname{var}(\hat{\alpha})
\end{array}\right]^{-1} .
$$

\subsection{Bayesian Estimation Method}

In this subsection, Bayesian estimation (BE) approach is used to estimate the parameters $\theta$ and $\alpha$ which are assumed to be independent and follow gamma prior distribution with parameters $a$ and $b$.

The gamma prior density function has the form

$$
g(u ; a, b)=\frac{b^{a}}{\Gamma(a)} u^{a-1} \mathrm{e}^{-u b}, \quad u, a, b>0 .
$$

Then, the joint prior density of $\theta$ and $\alpha$ is given by

$$
g(\theta, \alpha)=\prod_{i=1}^{n} g(\theta) g(\alpha) \propto(\theta \alpha)^{a-1} \mathrm{e}^{-(\theta+\alpha) b} .
$$

The joint posterior distribution function according to Bayesian procedure is 
given by

$$
g(\theta, \alpha \mid \underline{x})=\frac{g(\theta, \alpha) L(\underline{x})}{\int g(\theta, \alpha) L(\underline{x})} \propto g(\theta, \alpha) L(\underline{x}) .
$$

Substituting from Equations (28) and (18) into Equation (29) we get

$$
g(\theta, \alpha \mid \underline{x}) \propto(\theta \alpha)^{a-1} \mathrm{e}^{-\left(\theta\left(\sum_{i=1}^{n} x_{i}^{\alpha}+b\right)+\alpha b\right)} \prod_{i=1}^{n} x_{i}^{\alpha-1}\left(1+x_{i}^{\alpha}\right)^{3} .
$$

Markov Chain Monte Carlo method (MCMC) [2] is used to summarize the posterior distribution numerically without calculating the normalized constant.

\section{Applications and Goodness of Fit}

In this section, the goodness of fit of generalized power Akshaya distribution to real lifetime data is proposed and compared with some one parameter and two parameters distributions.

The data set represents the waiting times (in minutes) before service of 100 bank customers and analyzed and examined by Ghitany et al. [6] for fitting the lindley distribution. The data set is given as follows

Some statistics like, $-2 \ln (L)$, Akaike Information Criterion (AIC), Kolmogorov-Samirnov Statistics (K-S) and Bayesian estimate (BE) for this data are computed to compare between various lifetime distributions. These statistics are shown in Table 1.

The best distribution fitting the data is the distribution with least $-2 \ln (L)$, AIC, and least K-S statistics and Table 1 showed that power Akshaya distribution is better than others. The inverse of the information matrix of power Akshaya distribution using the estimated parameters, $\hat{\theta}$ and $\hat{\alpha}$ according to the MLE method can be given by

$$
F^{-1}=\left[\begin{array}{cc}
\operatorname{var}(\hat{\theta}) & \operatorname{cov}(\hat{\theta}, \hat{\alpha}) \\
\operatorname{cov}(\hat{\alpha}, \hat{\theta}) & \operatorname{var}(\hat{\alpha})
\end{array}\right]^{-1}=\left[\begin{array}{cc}
0.004 & -v e \\
-v e & 0.002
\end{array}\right]^{-1},
$$

\begin{tabular}{|c|c|c|c|c|c|c|c|c|}
\hline \multirow{2}{*}{ Distributions } & \multicolumn{2}{|c|}{ MLE } & \multicolumn{2}{|c|}{$\mathrm{BE}$} & \multirow{2}{*}{$-2 \ln (L)$} & \multirow{2}{*}{ AIC } & \multirow{2}{*}{ K-S } & \multirow{2}{*}{ P-value } \\
\hline & $\hat{\theta}$ & $\hat{\alpha}$ & $\hat{\theta}$ & $\hat{\alpha}$ & & & & \\
\hline power Akshaya & 0.556 & 0.814 & 0.559 & 0.813 & 635.73 & 639.73 & 0.04 & 0.9876 \\
\hline power Lindley & 0.153 & 1.083 & 0.160 & 1.072 & 636.64 & 640.64 & 0.50 & $2.2 \mathrm{e}^{-16}$ \\
\hline Akshaya & 0.368 & - & 0.368 & - & 649.72 & 651.72 & 0.13 & 0.074 \\
\hline Akash & 0.295 & - & 0.296 & - & 641.93 & 643.91 & 0.10 & $2.2 \mathrm{e}^{-16}$ \\
\hline Lindley & 0.187 & - & 0.187 & - & 638.07 & 640.07 & 0.058 & $2.2 \mathrm{e}^{-16}$ \\
\hline Exponential & 0.101 & - & 0.102 & - & 658.04 & 660.04 & 0.163 & $2.2 \mathrm{e}^{-16}$ \\
\hline
\end{tabular}

and the $95 \%$ confidence interval for the parameters $\theta$ and $\alpha$ can be given as

Table 1. The MLE, BE estimates, $-2 \ln (L)$, AIC, K-S and P-value statistics. 


$$
\left(\hat{\theta}_{L}, \hat{\theta}_{U}\right)=(0.431,0.680), \quad\left(\hat{\alpha}_{L}, \hat{\alpha}_{U}\right)=(0.719,0.908) .
$$

\section{Simulation Study}

In this section, random data of generalized power Akshaya is generated using the inverse of cumulative distribution function numerically. Mathematica program is used to generate different samples of the distribution when the size is $n$ $=20,50,70,100$, and 150 . The experiment is repeated 5000 times with initial values $\theta=1.5$ and $\alpha=0.5$. Five quantities are examined in this study

(a) Mean of the estimated values (ME) of $\hat{v}, \hat{v}=\hat{\theta}, \hat{\alpha}$ which equals $\frac{1}{5000} \sum_{i=1}^{5000} \hat{v}_{i}$.

(b) Average bias of the MLE (AB) of $\hat{v}$ which equals $\frac{1}{5000} \sum_{i=1}^{5000}\left(\hat{v}_{i}-v\right)$.

(c) The mean squared error (MSE) of the MLE of $\hat{v}$ which equals $\frac{1}{5000} \sum_{i=1}^{5000}\left(\hat{v}_{i}-v\right)^{2}$.

(d) Average width (AW) of $95 \%$ confidence intervals of parameter $v=\theta, \alpha$ which equals $(\hat{v}+1.96 v)-(\hat{v}-1.96 v)$.

(e) Coverage probability (CP) of $95 \%$ confidence intervals of parameter $v=\theta, \alpha$, i.e. the percentage of intervals that contain true values of the parameter $v$.

Table 2 shows that

- The absolute value of the average bias $|A B|$ for the parameters $\theta, \alpha$ decreases as the sample size $(n)$ increases.

- The mean squared error (MSE) for the parameters $\theta$ and $\alpha$ decreases as the sample size $(n)$ increases.

- The average width (AW) for the parameters $\theta$ and $\alpha$ decreases as the sample size $(n)$ increases.

Figure 5 shows the scaled TTT-transform, and it found increasing, and the empirical pdf for the simulated data. Figure 6 shows the Q-Q plots for the simulated data and distributions mentioned in Table 1 and it shows that the generalized power Akshaya distribution is the best fit for the data. Figure 7 shows the Kaplan Meier curve for the simulated data and the survival functions of the

Table 2. Some measures of the simulated data for various sample sizes.

\begin{tabular}{cccccccccccc}
\hline & \multicolumn{9}{c}{$\theta$} & \multicolumn{9}{c}{$\alpha$} \\
\cline { 2 - 10 } & ME & AB & MSE & AW & CP & ME & AB & MSE & AW & CP \\
\hline 20 & 1.478 & -0.022 & 0.053 & 0.881 & 0.943 & 0.532 & 0.032 & 0.009 & 0.320 & 0.946 \\
50 & 1.478 & -0.022 & 0.020 & 0.556 & 0.941 & 0.514 & 0.014 & 0.003 & 0.195 & 0.953 \\
70 & 1.487 & -0.013 & 0.014 & 0.471 & 0.951 & 0.511 & 0.011 & 0.002 & 0.165 & 0.955 \\
100 & 1.487 & -0.013 & 0.009 & 0.394 & 0.955 & 0.510 & 0.010 & 0.001 & 0.137 & 0.958 \\
150 & 1.488 & -0.012 & 0.007 & 0.322 & 0.951 & 0.508 & 0.008 & 0.001 & 0.112 & 0.946 \\
\hline
\end{tabular}



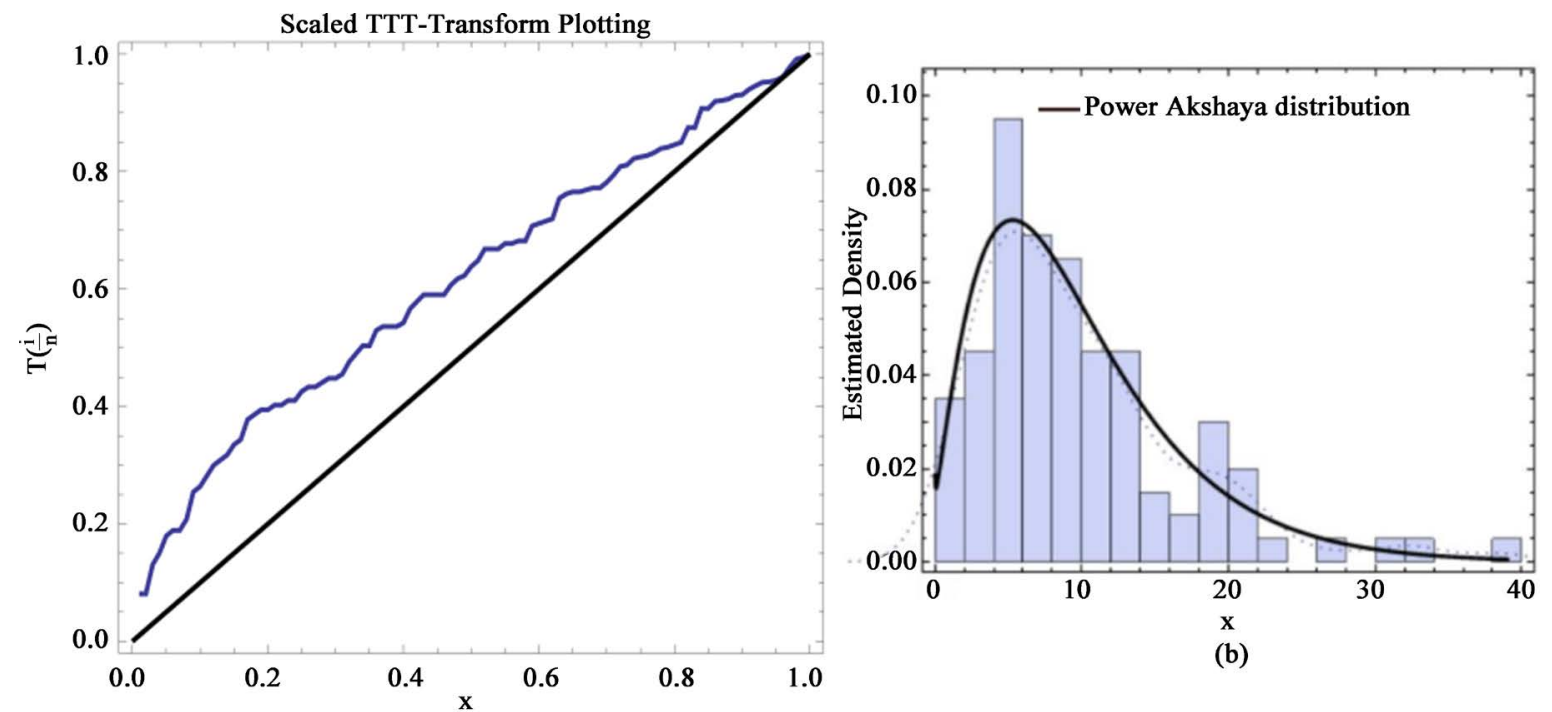

(a)

Figure 5. Scaled TTT-transform (a) and histogram (b) plots.
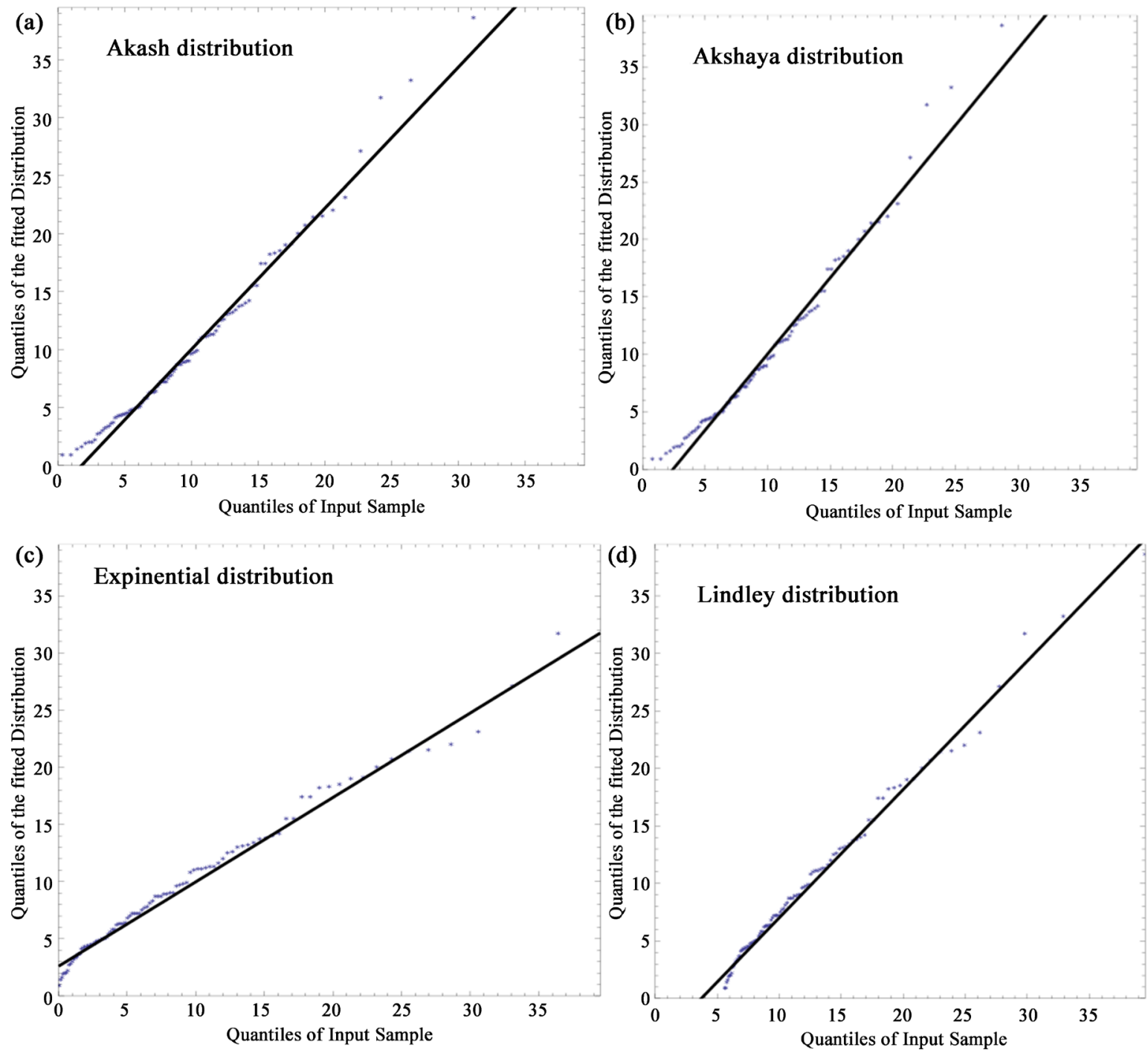

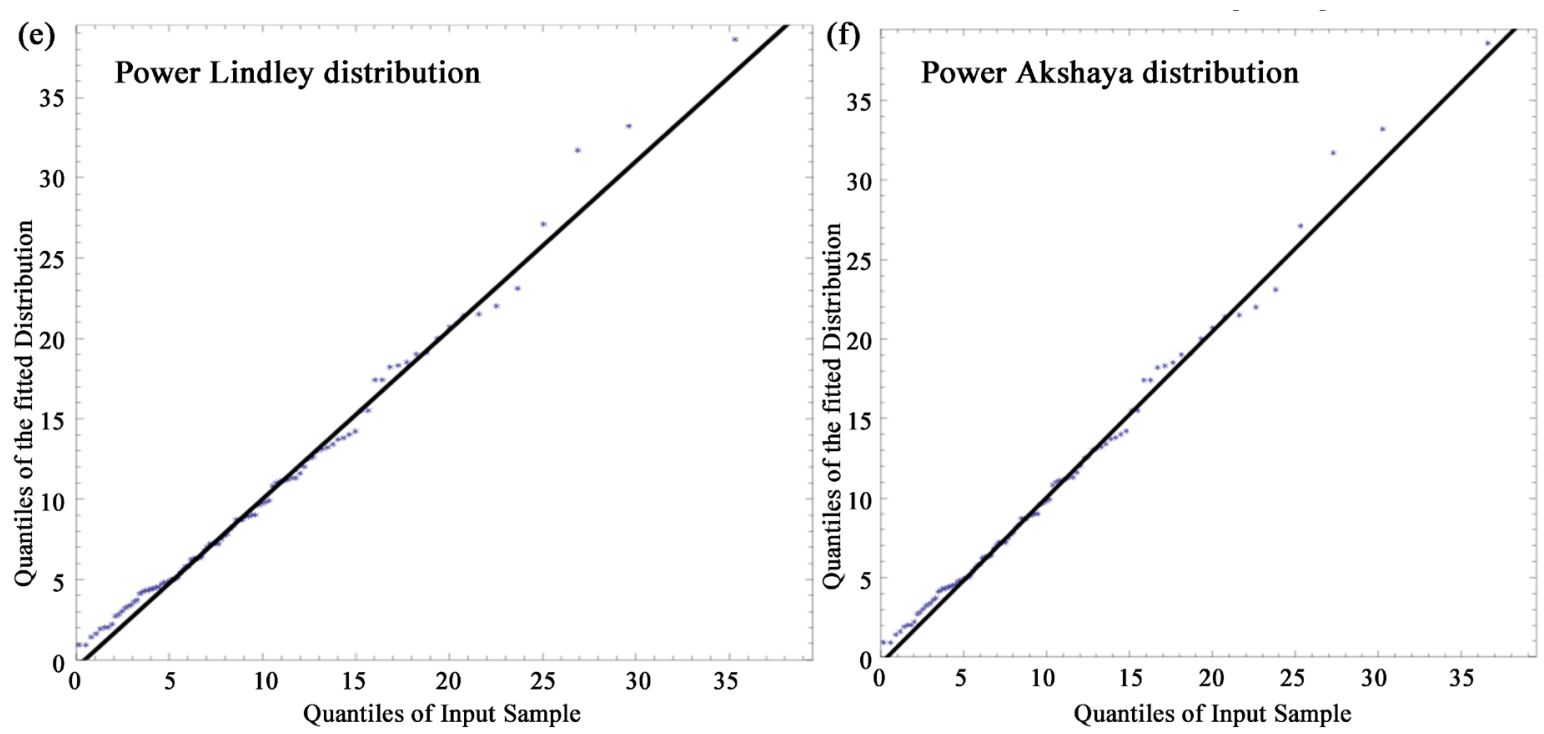

Figure 6. Q-Q plots for the simulated data.

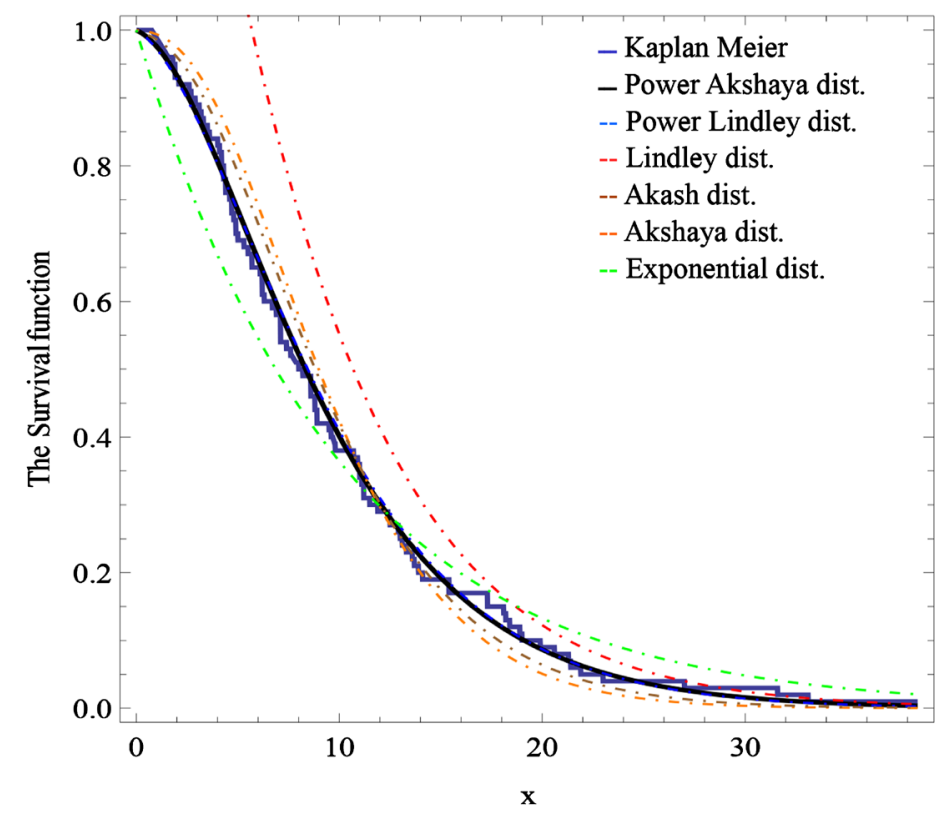

Figure 7. Kaplan Meier curve for the simulated data and the survival functions of some distributions.

Table 3. Posterior summaries for the simulated data.

\begin{tabular}{cccccccc}
\hline Prior & Parameters & Mean & SD & MC Error & $2.5 \%$ & Median & $97.5 \%$ \\
\hline Gamma & $\alpha$ & 0.8129 & 0.05851 & 0.002007 & 0.7201 & 0.8103 & 0.9091 \\
Gamma & $\theta$ & 0.559 & 0.06751 & 0.001758 & 0.4408 & 0.5589 & 0.6887 \\
\hline
\end{tabular}

distributions mentioned in Table 1 and also shows that the generalized power Akshaya distribution is the best fit for the data.

Table 3 shows a summary of some measures for the joint posterior distribution for the simulated data. Figure 8 and Figure 9 show the density and trace plot of parameters $\theta$ and $\alpha$ to assess the convergence visually. 

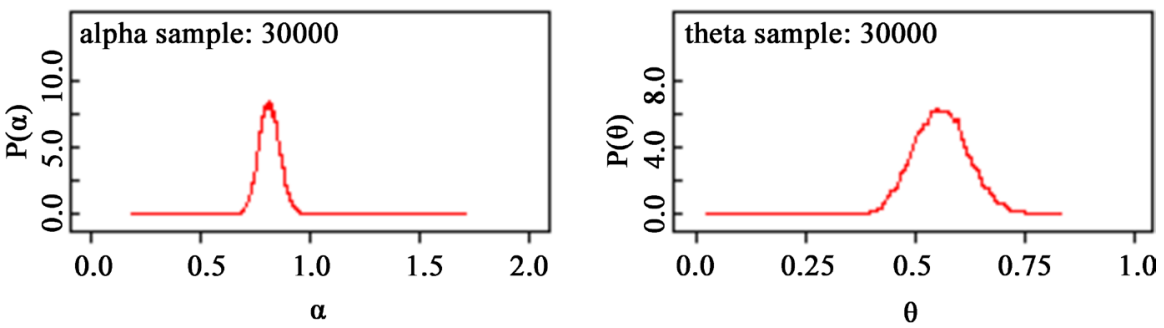

Figure 8. Marginal posterior density plots for the simulated data.
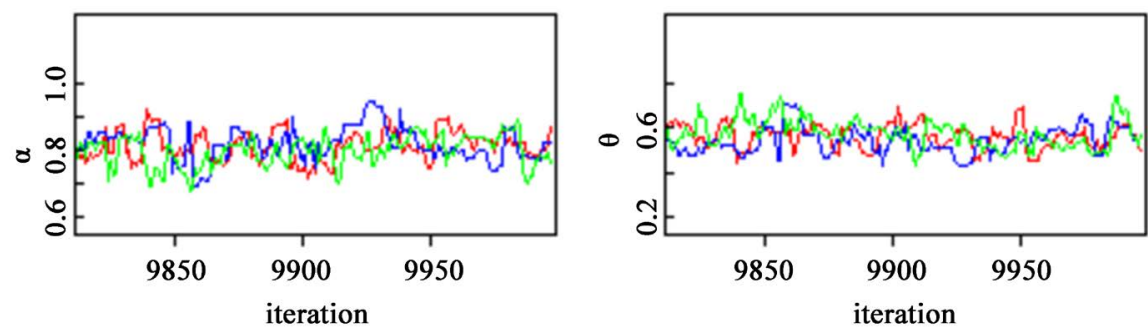

Figure 9. Trace plots for the simulated data.

\section{Conclusion}

A new two parameters lifetime distribution named generalized power Akshaya distribution has been introduced for modeling lifetime data. Some statistical properties such as cumulative distribution, density, survival, hazard and moments functions. Also, maximum likelihood and Bayesian techniques are used to estimate distribution parameters. The goodness of fit using $-2 \ln (L)$, Akaike Information Criterion (AIC), Kolmogorov-Samirnov Statistics (K-S) and P-value for real lifetime data have been presented to show its applicability overpower Lindley, Akshaya, Akash, Lindley and exponential distributions. Finally, a simulation study is carried out to show the mean of the estimated values. The average bias and mean square error of the maximum likelihood estimators of the model parameters are discussed. In addition, the coverage probability and average width of the confidence intervals for the parameters are calculated.

\section{Conflicts of Interest}

The authors declare no conflicts of interest regarding the publication of this paper.

\section{References}

[1] Al-Mofleh, H., Afify, A.Z. and Ibrahim, N.A. (2020) A New Extended Two-Parameter Distribution: Properties, Estimation Methods, and Applications in Medicine and Geology. Mathematics, 8, 15-78. https://doi.org/10.3390/math8091578

[2] Brooks, S. (1998) Markov Chain Monte Carlo Method and Its Application. Journal of the Royal Statistical Society: Series D (the Statistician), 47, 69-100. https://doi.org/10.1111/1467-9884.00117

[3] Eliwa, M.S., El-Morshedy, M. and Ali, S. (2020) Exponentiated Odd Chen-G Family of Distributions: Statistical Properties, Bayesian and Non-Bayesian Estimation with Applications. Journal of Applied Statistics, 2, 1-27. 
https://doi.org/10.1080/02664763.2020.1783520

[4] Epstein, B. and Sobel, M. (1953) Life Testing. Journal of the American Statistical Association, 48, 486-502. https://doi.org/10.1080/01621459.1953.10483488

[5] Ghitany, M.E., Al-Mutairi, D.K., Balakrishnan, N. and Al-Enezi, L.J. (2013) Power Lindley Distribution and Associated Inference. Computational Statistics \& Data Analysis, 64, 20-33. https://doi.org/10.1016/j.csda.2013.02.026

[6] Ghitany, M.E., Atieh, B. and Nadarajah, S. (2008) Lindley Distribution and Its Application. Mathematics and Computers in Simulation, 78, 493-506. https://doi.org/10.1016/j.matcom.2007.06.007

[7] Glaser, R.E. (1980) Bathtub and Related Failure Rate Characterizations. Journal of the American Statistical Association, 75, 667-672. https://doi.org/10.1080/01621459.1980.10477530

[8] Groeneveld, R.A. and Meeden, G. (1984) Measuring Skewness and Kurtosis. Journal of the Royal Statistical Society. Series D (The Statistician), 33, 391-399.

[9] Moors, J.J.A. (1988) A Quantile Alternative for Kurtosis. Journal of the Royal Statistical Society. Series D (The Statistician), 37, 25-23. https://doi.org/10.2307/2348376

[10] Nadarajah, S., Bakouch, H.S. and Tahmasbi, R. (2011) A Generalized Lindley Distribution. Sankhya B, 73, 331-359. https://doi.org/10.1007/s13571-011-0025-9

[11] Okasha, H. and Mustafa, A. (2020) E-Bayesian Estimation for the Weibull Distribution under Adaptive Type-I Progressive Hybrid Censored Competing Risks Data. Entropy, 22, 9-30. https://doi.org/10.3390/e22080903

[12] Shanker, R. (2015) Akash Distribution and Its Applications. International Journal of Probability and Statistics, 4, 65-75.

[13] Shanker, R. (2017) Akshaya Distribution and Its Application. American Journal of Mathematics and Statistics, 7, 51-59.

[14] Shanker, R., Hagos, F. and Sujatha, S. (2015) On Modeling of Lifetimes Data Using Exponential and Lindley Distributions. Biometrics \& Biostatistics International Journal, 2, 1-9. https://doi.org/10.15406/bbij.2015.02.00042

[15] Smith, R.L. and Naylor, J.C. (1987) A Comparison of Maximum Likelihood and Bayesian Estimators for the Three-Parameter Weibull Distribution. Journal of the Royal Statistical Society: Series C (Applied Statistics), 36, 358-369. 